\title{
Neutalizants for Antimicrobial Effective Preservatives in Microbiological Analysis in Cosmetic Products
}

\author{
Gökhan Cengiz, Evren Alğın Yapar* \\ Republic of Turkey Ministry of Health, Turkish Medicines And Medical Devices Agency, Vice Presidency of \\ Economic Assessments and Laboratory Services, Department of Analysis and Control Laboratories, 06100 \\ Sihhiye, Ankara, Turkey
}

*Correspondence: evrenalgin@yahoo.com (Evren Alğın Yapar)

Received: 07 November 2018; Accepted: 26 December 2018; Published: 31 December 2018

\begin{abstract}
Blue and green cosmetics are sensitive products for microbial contamination and reducing the contamination producing according to Cosmetic Good Manufacturing Practice (ISO 22716), providing proper storage condition and packaging are effective factors. In addition antimicrobial effective preservatives can be added to protect the cosmetic products. ${ }^{1,2}$ Generally, preservatives are chemical substrates that are effective on cytoplasm (conjugation mechanism, ribosomes, nucleic acids, thiol groups, amino groups), cell wall and cytoplasm membrane (membrane potential, enzymes, membrane penetration). ${ }^{3}$ The neutralization of the antimicrobial effect of the preservatives in microbiological quality control of cosmetics is essential for the safety of the tests. The antimicrobial preservative in the cosmetic which is transferred to the medium affects the test negatively. The neutralization process can be carried out according to international standards used in microbiological analysis of cosmetic products. The neutralization of preservatives in cosmetics can be performed by adding of neutralizing agents in solvent, medium and/or washing solution. The agents which are reacted with the preservative groups for the removal of antimicrobial affect are used for this neutralization process which is necessary for the removal of antimicrobial effect. According to European Pharmacopeia 2.6.12. sodium bisulfite for glutaraldehyde and mercury compounds; dilution for sorbates and aldehydes; lecithin, polysorbate 80 , saponin for quaternary ammonium compounds, parabens and iodines; thioglycollate for mercurial; thiosulfate for halogens can be used as neutralizing agents. ${ }^{4}$ Lecithin, polysorbate 80 for parabens, phenoxyethanol, phenylethanol; Lecithin, saponin, polysorbate 80 , sodium dodecyl sulphate for quaternary ammonium compounds and cationic surfactants; glycine, histidine for aldehydes formaldehyde-release agents; sodium thiosulphate for oxidizing agents; sodium bisulphate, L-cysteine and thioglycollic acid for metallic salts can be added to the medium, solvent and/or washing solutions according to ISO $21149 .{ }^{5}$ For the removal of antimicrobial effect, neutralizing agents in proper concentrations should be added to medium, solvents and washing solutions. The addition of these agents with antimicrobial effect should be proved by the studies of conformity. Neutralizing agents react with antimicrobial agents to eliminate the antimicrobial effect.
\end{abstract}

Key Words: Cosmetic product, Microbiological control, Neutralization, Neutralizing agents

\section{Introduction}

Blue and green cosmetics are sensitive products for microbial contamination. Therefore, their production according to Cosmetic Good Manufacturing Practice (ISO 22716 ) is effective in decreasing of contamination by proper storage condition and packaging and antimicrobials can be added for preservation, additionaly. Generally, preservatives are chemical substrates that are effective on cytoplasm (conjugation mechanism, ribosomes, nucleic acids, tiol groups, amino groups), cell wall and cytoplasm 
membrane (membrane potential, enzymes, membrane penetration). The neutralization of the antimicrobial effect of the preservatives in microbiological quality control of cosmetics is essential for the safety of tests. The antimicrobial preservative in the cosmetic which was transferred to the medium affects the test in a negative way. Neutralization process is performed according to the international standards used in microbiological analyses of cosmetics. The neutralization of the preservatives in cosmetics can be performed by adding of neutralizing agents in solvent, medium and/or washing solution. The agents which are reacted with the preservative groups for the removal of antimicrobial affect are used for this neutralization process which is necessary for the removal of antimicrobial effect.

\section{Methods}

In microbiological quality control tests, antimicrobial agents should be neutralized. It is done according to EP 2.6.12, USP 61 or ISO 21149 standards for neutralization process. According to these standards, neutralizers are added to the medium, solvent and / or washing solutions. These neutralizers to be added vary according to the antimicrobial preservative group. For the removal of antimicrobial effect, neutralizing agents in proper concentrations should be added on medium, solvents and washing solutions. Added agents which do not have any antimicrobial effect should be proved with method suitability studies. The neutralizing agents are used for the removal of antimicrobial effect by reacting with antimicrobial effective chemicals. The neutralizers which should be used according to the preservative are shown in the tables.

Table.1 Neutralizers for antimcrobial preservative

\begin{tabular}{|c|c|}
\hline Antimicrobial Agents & Neutralizers (EP 2.6.12 and USP 61) \\
\hline Glutaraldehyde, mercurials & Sodium hydrogen sulfite (Sodium bisulfite) \\
\hline Phenolics, alcohol, aldehydes, sorbate & Dilution \\
\hline Aldehydes & Glycine \\
\hline $\begin{array}{l}\text { Quaternary ammonium compounds (QACs), } \\
\text { parahydroxybenzoates (parabens), } \\
\text { bis-biguanides }\end{array}$ & Lecithin \\
\hline QACs, iodine, parabens & Polysorbate \\
\hline Mercurials & Thioglycollate \\
\hline Mercurials, halogens, aldehydes & Thiosulfate \\
\hline EDTA (edetate) & Mg or Ca ions \\
\hline
\end{tabular}

Table.2 Neutralizers for antimcrobial preservative

\begin{tabular}{ll} 
Antimicrobial Agents & Neutralizers (EP 2.6.12 and USP 61) \\
\hline Parabens, Phenoxyethanol, Phenylethanol, & $\begin{array}{l}\text { Lecithin, Polysorbate 80, Ethylene oxide } \\
\text { condensate of fatty alcohol, Non-ionic } \\
\text { surfactants } \\
\text { Lecithin, saponin, polysorbate 80, sodium } \\
\text { dodecyl sulphate }\end{array}$ \\
Quaternary ammonium compounds & $\begin{array}{l}\text { Glycine, histidine } \\
\text { Aldehydes }\end{array}$ \\
Sodium thiosulphate \\
Biguanides & $\begin{array}{l}\text { Lecithin, saponin, polysorbate 80 } \\
\text { Metallic salts (Cu, Zn, Hg) }\end{array}$ \\
Organo-mercuric compounds & Sulfhydryl compounds, thioglycollic acid \\
Isothiazolinones, imidazoles & Lecithin, saponin \\
\hline
\end{tabular}




\section{Results and Discussion}

For the method suitability study, the product is tested with 5 microorganisms of a specific concentration and compared to the number of microorganisms recovered from the control preparation. If growth is inhibited (reduction by a factor greater than 2), then modify the procedure for the particular enumeration test to ensure the validity of the results. Modification of the procedure may include, for example, an increase in the volume of the diluent or culture medium; incorporation of a specific or general neutralizing agents into the diluent; membrane filtration; or acombination of the above measures. If no suitable neutralizing method can be found, it can be assumed that the failure to isolate the inoculated organism is attributable to the microbicidal activity of the product. This information serves to indicate that the article is not likely to be contaminated with the given species of the microorganism. However, it is possible that the product inhibits only some of the microorganisms specified herein, but does not inhibit others not included among the test strains or those for which the latter are not representative. Then, perform the test with the highest dilution factor compatible with microbial growth and the specific acceptance criterion.

As a result of the neutralization process, the recovery must comply with the acceptance criteria. Alternative neutralization methods should be applied in unsuitable results. In alternative methods applied, appropriate results should be obtained according to the acceptance criteria. The neutralization of antimicrobial preservatives in cosmetic products is important in terms of test reliability. Neutralization of antimicrobial activity should be demonstrated in the quality control tests to be applied. The results should be appropriate according to the acceptance criteria.

\section{References}

Republic Turkey Ministry of Health. Turkish Medicines And Medical Devices Agency Cosmetic Law, 2005, No, 25823.

ISO 22716, Cosmetics - Good Manufacturing Practices (GMP) - Guidelines on Good Manufacturing Practices, 2013.

ISO 17516, Cosmetic microbiology microbiological limits, 2014.

European Pharmacopoeia 9.0, 2.6.12. Microbiological exaimination of non-sterile products: microbial enumeration tests, 2017.

ISO 21149, Cosmetic microbiology enumeration and detection of aerobic mesophilic bacteria, 2006. 